\title{
Prescribing costs in dispensing practices
}

\author{
Tony J Morton-Jones, Mike A L Pringle
}

Abstract

Objective-To examine differences in prescribing between dispensing and non-dispensing practices.

Setting-The 108 practices covered by Lincolnshire Family Health Services Authority.

Design-Analysis of prescribing data for 1990-1 from PD2 reports from the Prescription Pricing Authority in relation to data on practice characteristics obtained from Lincolnshire Family Health Services Authority; and aggregated level 3 prescribing and cost information (PACT data) for 10 selected drugs from the Prescription Pricing Authority to examine amounts prescribed.

Main outcome measures-Prescribing cost per patient, items per patient, and cost per item in dispensing and non-dispensing practices.

Results-Dispensing practices had higher prescribing costs per patient than non-dispensing practices. This difference held for non-dispensing patients within dispensing practices. Structural features failed to explain the differences in prescribing cost, except for the higher numbers of elderly patients in dispensing practices (which explained $13 \%$ of the difference) and the number of partners $(5 \%)$. The main determinant of the difference was the lower use of generic drugs in dispensing practices $(84 \%)$. Dispensing patients were prescribed lower quantities of drugs on average for each item.

Conclusions-Dispensing practices could reduce their prescribing expenditure to that of nondispensing practices by increasing their prescribing of generic drugs. The shorter prescribing intervals for dispensing patients may be due to dispensing fees being related to the number of prescribed items.

\section{Introduction}

All general practitioners prescribe, and most of their patients take their prescriptions to a pharmacy. A minority, however, take their prescriptions to a dispensary within the practice. In $1989-90$ the $14 \%$ of general practitioners who dispensed drugs ${ }^{1}$ made up 27013820 prescriptions $(6 \cdot 75 \%$ of all primary care prescriptions). ${ }^{2}$

The need for doctors to dispense arose in rural areas to compensate for the absence of pharmacies. Dispensing practices can dispense only for patients who live more than one mile from a pharmacy, so most dispense for only some of their patients.

The rural setting of most dispensing practices means that their geographical distribution is uneven. The five family health service authorities with the highest percentage of dispensing practitioners are Lincolnshire $(52 \%)$, North Yorkshire (52\%), Cambridgeshire (48\%),

Department of General Practice, Queen's Medical Centre, Nottingham NG7 2UH

Tony J Morton-Jones, research fellow

Mike A L Pringle, senior lecturer

Correspondence to: Dr Pringle.

BMf 1993;306:1244-6 comparison between dispensing and non-dispensing practices in Lincolnshire.

\section{Methods}

Three sources of data were used. Firstly, prescribing data (PD2) from the Prescription Pricing Authority were obtained for all $108 \mathrm{Lincolnshire} \mathrm{practices.} \mathrm{These}$ data covered 1990-1 and distinguished between prescriptions issued to dispensing and non-dispensing patients. This allowed three categories of prescriptions to be derived: those issued to patients in nondispensing practices; those to non-dispensing patients in dispensing practices; and those to dispensing patients in dispensing practices. The prescribing variables used were the average number of items per patient, the average net ingredient cost per item, the average net ingredient cost per patient, and the percentage of items prescribed generically.

Secondly, data on each practice were obtained from Lincolnshire Family Health Services Authority concerning the number of partners, registered list characteristics, rurality, pharmacy location, underprivileged area (Jarman) score, and appointment systems. Rurality was defined as the mean distance (in round kilometres with distances of less than a kilometre being rounded up to one) between patients' homes and the practice premises; the pharmacy location was the distance between the nearest pharmacy and the practice; the Jarman score was calculated by endowing all patients with the score for their ward of residence and averaging the scores for patients registered with the practice. The practices were grouped into those offering only appointments surgeries and those with either a mixture or only open surgeries. Since these data related to entire practices we could compare only dispensing and non-dispensing practices and not the patients within them.

The third data source was the aggregated level 3 prescribing and cost information from the Prescription Pricing Authority (PACT data) for the whole of Lincolnshire covering January to March 1990. From this the prescribing of 10 selected drugs (see table II) was extracted. These data were divided into two groups-prescriptions to dispensing patients and those to non-dispensing patients (from all practices) since it was not possible to distinguish between non-dispensing patients of dispensing practices and patients of non-dispensing practices. The 10 drugs were selected because each had a single specific clinical indication and were usually used for long term treatment and there was no clinical reason to expect any differences between dispensing and non-dispensing practices.

The prescribing cost per patient, items per patient, and cost per item averages were computed by using the total number of items, list size, and net ingredient cost for the group as a whole-that is, aggregated over the practices within the group. The data in the analysis of variance and multiple regression analysis used the averages for each practice, weighted by the ratio of practice list size to the average list size. This allowed the differences in costs between dispensing practices and non-dispensing practices to be equivalent in the analyses. The prescribing data were analysed by using general, ${ }^{3-6}$ with none specifically on prescribing in dispensing practices. ${ }^{4}$ This is despite the fact that dispensing practices issue more prescriptions per patient at a greater average cost per patient. ${ }^{178}$ Our study examined the influences on prescribing in a 
analysis of variance for comparison of means among the three categories of patients. Analysis of variance produced average values for net ingredient cost per patient, net ingredient cost per item, and items per patient for differing groups of practices or patients, the groups being defined by dispensing status or by the value of a practice factor (rurality, generics, etc). The analysis tested for any significant differences.

The differences in practice characteristics and the amount of generic prescribing between dispensing and non-dispensing practices were assessed by Student's $t$ test. The effect of practice characteristics and generic prescribing on prescribing rates and costs was also assessed through analysis of variance. Multiple regression was used to fit (through a least squares procedure) the average net ingredient cost per patient for each practice with practice factors. The analysis generated regression coefficients which gave the dependence of net ingredient cost per patient on each factor. Using these and the average values of each factor for dispensing and non-dispensing practices we were able to estimate the predicted difference in net ingredient cost per patient between dispensing and non-dispensing practices with a contribution from each significant factor. In the analysis the net ingredient cost per patient was used as the dependent variable and initially all practice characteristics (including dispensing status) and percentage generics were used as independent variables. The significant independent variables were found by the forward selection procedure. None of these were eliminated on the backward selection procedure.

\section{Results}

The average annual net ingredient cost per patient for non-dispensing practices was $£ 48.47$, compared with $£ 54.78$ for dispensing practices $(t=-3 \cdot 2$, $p=0.002)$. The differences shown in table $I$ were determined by practice type (non-dispensing or dispensing) rather than patient type. However, the number of items per patient and the cost of each item also differed with patient type. The dispensing patients had more and cheaper items than the other two groups, while the non-dispensing patients in dispensing practices had fewer items than the dispensing patients but more expensive ones. Overall, dispensing patients received $14 \%$ more items than non-dispensing patients.

To assess whether this difference was due to the

TABLE —Prescribing variables for different patient-practice groupings

\begin{tabular}{|c|c|c|c|}
\hline Patient group & $\begin{array}{c}\text { Annual } \\
\text { net ingredient cost } \\
\text { per patient }\end{array}$ & $\begin{array}{c}\text { Annual } \\
\text { net ingredient cost } \\
\text { per item }\end{array}$ & $\begin{array}{c}\text { Annual } \\
\text { No of items per } \\
\text { patient }\end{array}$ \\
\hline $\begin{array}{l}\text { Patients in non-dispensing practices } \\
\text { Patients in dispensing practices }\end{array}$ & $\begin{array}{l}48 \cdot 47^{\star} \\
54 \cdot 78\end{array}$ & $\begin{array}{l}5 \cdot 83 \\
5 \cdot 74\end{array}$ & $\begin{array}{l}8 \cdot 32^{\star} \\
9 \cdot 55\end{array}$ \\
\hline $\begin{array}{l}\text { Non-dispensing patients } \\
\text { Dispensing patients }\end{array}$ & $\begin{array}{l}55 \cdot 13 \\
54 \cdot 53\end{array}$ & $\begin{array}{l}6 \cdot 07 \\
5 \cdot 49^{\star}\end{array}$ & $\begin{array}{l}9.09 \\
9.93\end{array}$ \\
\hline All patients & 51.76 & $5 \cdot 78$ & 8.96 \\
\hline
\end{tabular}

*Group value significantly different $(p<0.05, \mathrm{df}=107)$ from the other two patient classes (least squares difference procedure from analysis of variance).

TABLE I-Average quantity of drugs prescribed per prescription for non-dispensing patients (in all practices) and dispensing patients (quarter ending March 1990) for 10 selected drugs

\begin{tabular}{lcccc}
\hline Drug (and unit prescribed) & $\begin{array}{c}\text { Section in } \\
\text { PACT }\end{array}$ & $\begin{array}{c}\text { Non-dispensing } \\
\text { patients }\end{array}$ & $\begin{array}{c}\text { Dispensing } \\
\text { patients }\end{array}$ & $\begin{array}{c}\% \\
\text { Difference }\end{array}$ \\
\hline Glibenclamide (5 mg) & 6.1 .2 .1 & 74 & 63 & 17 \\
Atenolol (100 mg) & 2.4 & 41 & 34 & 21 \\
Allopurinol (300 mg) & 10.1 .4 & 41 & 34 & 21 \\
Bendrofluazide (5 mg) & 2.2 .1 & 43 & 38 & 13 \\
Aspirin (75 mg) & 2.9 & 52 & 45 & 16 \\
Adalat Retard (nifedipine 20 mg) & 2.6 .2 & 74 & 69 & 7 \\
Voltarol Retard (diclofenac 100 mg) & 10.1 .1 & 35 & 31 & 12 \\
Frumil (amilionide 5 mg, frusemide 40 mg) & 2.2 .4 & 44 & 39 & 14 \\
Temazepam (20 mg) & 4.1 .1 & 40 & 38 & 5 \\
Thyroxine (100 mg) & 6.2 .1 & 56 & 50 & 11 \\
\hline
\end{tabular}

TABLE III-Average values of practice characteristics for dispensing and non-dispensing practices in Lincolnshire

\begin{tabular}{lccc}
\hline Factor & $\begin{array}{c}\text { Dispensing } \\
\text { (59 practices) }\end{array}$ & $\begin{array}{c}\text { Non-dispensing } \\
\text { (49 practices) }\end{array}$ & Significance \\
\hline No of partners & 2.85 & 2.96 & NS \\
List size per GP & 1884 & 2009 & NS \\
Rurality (km) & 2.43 & 1.25 & $<0.001$ \\
Pharmacy location (km) & 4.36 & 1.0 & $\star$ \\
Jarman score & -5.43 & 5.48 & $<0.001$ \\
Appointment system (1 or 2) & 1.37 & 1.11 & 0.002 \\
Fraction of patients over 65 & 0.195 & 0.176 & $<0.001$ \\
\% Of generic drugs prescribed & 26.5 & 42.0 & $<0.001$ \\
& & & \\
\hline
\end{tabular}

*No variance in the non-dispensing practices, as all had a pharmacy nearer than $1 \mathrm{~km}$ from the practice.

TABLE IV-Contributions of significant factors to explaining prescribing cost differences between dispensing and non-dispensing practices

\begin{tabular}{lccc}
\hline Factor & $\begin{array}{c}\text { Regression } \\
\text { coefficient (SE) }\end{array}$ & $\begin{array}{c}\text { Contribution } \\
(£)\end{array}$ & $\begin{array}{c}\% \\
\text { Contribution }\end{array}$ \\
\hline \% Of generic drugs prescribed & $-0.34(0.07)$ & 5.33 & 84 \\
No of partners & $-1.60(0.42)$ & 0.33 & 5 \\
$\%$ Of patients aged $\geqslant 65$ & $94.82(19.14)$ & 0.85 & 13 \\
\hline
\end{tabular}

prescribing of more simple remedies to dispensing patients or to the prescribing of smaller quantities more often we analysed the data on 10 selected drugs for the whole of Lincolnshire. This showed that dispensing patients in dispensing practices received significantly lower quantities (on average $13 \cdot 7 \%$ lower) in each prescription than non-dispensing patients in both dispensing practices and non-dispensing practices (table II). The percentage of increased items for the selected drugs $(13.7 \%)$ was comparable to the overall percentage difference in items per patient between dispensing and non-dispensing patients $(14 \%)$.

Significant differences existed in the percentage of generic drugs prescribed, rurality, pharmacy location, Jarman score, appointment systems, and numbers of patients over 65 between dispensing and nondispensing practices (table III). The difference in numbers of partners and list size, although statistically insignificant, must still be considered because small differences in practice size may have a strong effect on cost.

The analysis of variance showed that rurality, pharmacy location, Jarman score, and appointment systems did not significantly explain the differences in prescribing costs between dispensing and nondispensing practices. Only the percentage of generic drugs prescribed, the proportion of patients over 65 , and the number of partners were significant.

The results of the multiple regression procedure tied in with the analysis of variance results. The final model retained (through statistical means) the number of partners, the proportion of patients over 65 , and the percentage of generic drugs prescribed as significant factors in determining average net ingredient costs per patient. The multiple regression correlation coefficient was 0.62 , and thus the model explained $38 \%$ of the variation in net ingredient costs per patient among all 108 practices. Use of the regression coefficients and the average value for each factor for all dispensing practices and all non-dispensing practices led to the contribution of each factor to the observed difference in average net ingredient cost per patient (table IV).

\section{Discussion}

Despite claims to the contrary, ${ }^{9}$ dispensing doctors are high cost prescribers. This study has shown that in Lincolnshire drug costs for dispensing practices are $£ 6.31$ per patient per year more $(13 \%)$ than for patients of non-dispensing practices. Furthermore, dispensing patients receive more, albeit cheaper, items than nondispensing patients. Using the average figures from 
table I and the Lincolnshire patient population of around 600000 , we can calculate the potential saving if all dispensing practices were to reduce their prescribing costs to that of the non-dispensing practices. The overall average net ingredient cost per patient would then be $£ 48.47$ (the average for non-dispensing practice patients), falling from the observed overall average of $£ 51.76$. Multiplying this difference by the number of patients, we arrive at a saving of $£(51.76-48.67) \times 600000=£ 1974000$ per year for Lincolnshire. It is important to understand the reasons for the differences to decide what portion of these savings can realistically be achieved.

A striking finding is that the cost per patient was similar for both non-dispensing and dispensing patients in dispensing practices. The determinant of cost is thus the dispensing status not of the patient but of the practice, indicating that practice characteristics may be important in explaining the cost differences.

Most practice characteristics, such as rurality and pharmacy location, however, have been shown in this study to be of no importance in explaining these differences. While the number of partners was retained in the final multiple regression model of net ingredient cost per patient, the difference in the average value between dispensing and non-dispensing practices was small, making the contribution to the cost difference only $5 \%$. The only important contribution from practice characteristics was from the higher numbers of patients over 65 , which explained $13 \%$ of the difference.

The main determinant of the difference, explaining $84 \%$, was associated with dispensing doctors' reluctance to prescribe generically. Several factors concerning generic prescribing are peculiar to dispensing practices. Many dispensing doctors are anxious about product liability legislation. ${ }^{10} 11$ If a drug is incorrectly formulated, for example, and this results in injury to a patient then the liability rests with the supplier (the dispensing practice) unless he can demonstrate his source satisfactorily (the wholesaler or manufacturer) and transfer the liability up the supply chain. Many dispensing doctors therefore perceive less risk with proprietary medicines than with generic drugs. In reality if a dispensing practice uses a single source for each drug or records batch numbers and manufacturers for each dispensed prescription no liability should reside in the practice.

Another complicating factor is the use of "named generics." Some drugs are available with a brand name at a generic price, and dispensing practices prefer these because they combine the extra security of proprietary prescribing with generic costs. In the returns from the Prescription Pricing Authority these drugs do not appear as part of the generic prescribing percentage, so these data obfuscate the true effect of generic drugs on prescribing costs. A comparative analysis of the use of brand name generics by dispensing and non-dispensing practices is therefore necessary. Even if branded generic drugs have some mitigating effect on the generic factor, however, the observed differences must still be explained by other factors.

The three factors in the final multiple regression model predict a total difference in prescribing costs of $£ 6.51$ per patient between dispensing and nondispensing practices-which agrees closely with the actual difference of $f 6.31$. The model is therefore effective in highlighting the origins of the differences in average net ingredient cost per patient between the two groups of practices. There is no reason to believe that similar differences are not manifested in other family health services authorities with significant numbers of dispensing practices as the factors will not be specific to Lincolnshire doctors. However, the model is less effective at predicting the individual practice prescribing costs, only $38 \%$ of the overall variation being explained. This is somewhat less than can be achieved with aggregated family health services authority data $^{312}$ and is most likely associated with individual practice prescribing philosophies and local factors peculiar to the practice, which give individual departures from the model. These practice differences do not appear to be significant when comparing dispensing and non-dispensing practice costs; their average effect must be the same for both groups.

The profitability of a dispensary is closely related to both the overall turnover of stock and the number of precriptions issued, since each prescription attracts a dispensing fee and container allowance. The number of prescriptions can be increased without affecting turnover by decreasing prescribing intervals. While there is no evidence to suggest that financial incentives increase turnover in dispensing practices (as their higher costs per patient are explicable in terms of the regression model), there is, however, evidence to suggest that financial incentives lead dispensing doctors to issue precriptions for shorter periods (tables I and II). The increased number of (cheaper) items prescribed to dispensing patients could be a result of the prescribing of "over the counter" substitutes, but the PACT 3 analysis shows that these patients have $13.7 \%$ more items of the 10 specific drugs examined, in line with $14 \%$ more items overall. Shorter prescribing intervals therefore appear to be the major contributor to the increased number of items prescribed, with precribing of simple remedies thus playing only a minor role. One burden of this increased number of items falls on the patient who pays prescription charges, whose costs will increase.

In summary, dispensing practices in Lincolnshire are relatively high cost prescribers. Their increased numbers of elderly patients explain some of the extra cost, but their prescribing a smaller proportion of generic drugs than other practices is the most significant factor, although their greater use of brand name generics may mitigate this. If brand name generics are important then other factors to explain the differences must be found. The financial incentives connected with shorter prescribing intervals seem to contribute to the higher numbers of cheaper items being prescribed.

We thank Lincolnshire Family Health Services Authority for funding this project; Jim Gallagher, Stuart Evans, Ian Wakeford, Martin Hardy, and Mark Anderson at Lincolnshire Family Health Services Authority for the provision of practice data and many useful discussions; Paul Dixon at the Centre for Health Economics, University of York, for providing a copy of the 1989-90 Health Services Indicator dataset; Valerie Peel and June Wakefield of the Prescription Pricing Authority for helpful advice and provision of PACT data and annual reports.

1 Health Services Indicator dataset 1989-90. London: Department of Health, 1991.

2 Prescription Pricing Authority. Annual repon 1 April 1990-31 March 1991. Newcastle: PPA, 1991:8.

3 Forster DP, Frost CEB. Use of regression analysis to explain the variation in prescribing rates and costs between family practitioner committees. $\mathrm{Br}$ Gen Pract 1991;41:67-71.

4 Gilleghan JD. Prescribing in general practice. London: Royal College of Generel Practitioners, 1991. (Occasional paper 54.)

5 Bradley CP. Decision making and prescribing patterns-a literature review. Family Practice 1991;8:276-87.

6 Harris CM, Heywood PL, Clayden AD. The analysis of prescribing in gevieral practice. London: HMSO, 1990.

7 Morton-Jones AJ, Pringle MAL. Dispensing doctors. BMJ 1992;305:478.

8 Geddes GL. Dispensing doctors. BMY 1992;305:478.

10 Dyer C. Strict product liability arrives: implications for doctors. BMO 1988;2\%:735.

11 Jennings SJ. Product liability and GPs. BMF 1988;296:936.

12 Morton-Jones AJ, Pringle MAL. Explaining variations in precribing costs across England. $B M Y$ (in press)

(Accepted 23 March 1993) 